ment chauffés dans des lacto-sérums d'où les complexes caséinophosphatiques du lait avaient été éliminés par emprésurage.

$$
*^{*} *
$$

L'ensemble des faits précédents tend évidemment à confirmer l'hypothèse de laquelle nous sommes partis en abordant ce travail. $\mathrm{Jls}$ permettent en outre de donner à cette hypothèse une forme plus précise.

On peut admettre qu'une réaction d'ordre physique ou chimique intervient entre les couches superficielles des membranes microbiennes ou les capsules qui les recouvrent d'une part, et d'autre part un élément colloïdal du milieu en l'espèce les phospho-caséinates. Cette réaction, amorcée déjà à la température ordinaire, s'exagérerait sous l'influence du chauffage et elle aboutirait ou non, suivant les cas, à la formation d'une enveloppe protectrice résistant à certains colorants. Et ce serait, en fin de compte, la nature des surfaces capsulaires mises en contact avec les phospho-caséinates, et par conséquent l'espèce et l'état cultural des souches microbiennes soumises à nos chauffages d'épreuves dans le lait, qui conditionneraient l'apparition de nos formes microbiennes protégées contre l'action du bleu de méthylène.

Mais on peut aussi expliquer les faits précédemment relatés par d'autres hypothèses et notamment par celle d'adsorbtions électives des colorants modifiées par l'action du chauffage.

\title{
BIBLTOGRAPHIE
}

[1] Gorini, Les acidoprotéolytiques dans la science et dans la pratique. Volume Jubilaire du Professeur Ch. Porcher.

[2] Ch. Porcher. Le lait au point de vue colloïdal. - Editeur : Le Lait.

[3] BonI. Ricerche sulla capsula dei Bacteri. Giorn. et Loc. ital. d'igiene, XXIII, 1901, p. 417.

\section{A PROPOS DE GARES LAITIÈRES}

\author{
par
}

\section{Le Docteur G. BARTHÉLEMI}

M.R.San.I

Médecin-hygiéniste

Laboratoire d'hygiène de l'Université de Bruxelles

Le transport du lait à longue distance constitue, dans certains cas, une nécessité pour assurer l'approvisionnement des grandes villes et pose un problème parfois fort important de manutention.

On ne peut, en effet, imaginer que des étables de vaches laitières soient installées dans l'enceinte des grandes agglomérations, de 
manière à ce que leur production réponde aux besoins de la consommation urbaine; production qui serait d'ailleurs loin d'être suffisante à moins de disposer d'un nombre considérable d'étables dont la place n'est certainement pas au milieu d'espaces bâtis. Les bêtes n'y jouissent notamment pas d'une atmosphère suffisamment pure, ni des herbages indispensables à leur bon équilibre nutritif. Ces deux facteurs influencent trop la production laitière pour qu'il n'en soit pas tenu compte.

Force est donc de s'adresser, pour la consommation élevée des grandes villes, à du lait provenant soit des environs immédiats des agglomérations, soit de régions plus éloignées, parfois distantes de 100 à $200 \mathrm{~km}$., et même davantage, du lieu d'utilisation.

Ces nécessités posent par conséquent le problème du transport du lait et impliquent le respect d'un certain nombre de conditions inhérentes à la fragilité même de la denrée transportée.

En ce qui concerne le lait de banlieue, le facteur temps n'impose pas, en réalité, de précautions particulières. Lorsque le lait est produit dans de bonnes conditions et qu'il est convenablement filtré et refroidi à la ferme aussitôt après la traite, il arrive chez le détaillant ou l'intermédiaire dans un état de fraîcheur encore suffisant, grâce à la commodité et à la rapidité du transport automobile. Ses qualités de conservation sont d'autant meilleures que le transport s'est effectué de nuit ou très tôt le matin, aux heures les moins chaudes de la journée. Les avantages sont encore plus marqués quand le transport à courte distance peut se faire en citernes calorifugées plutôt qu'en cruches.

On ne peut se faire d'illusion quant à la température du lait à son arrivée au centre de distribution quand les cruches ont été exposées, au soleil d'été et sans aucun moyen de protection, pendant toute la durée d'une tournée de ramassage.

Mais quand le lait provient de régions agricoles éloignées et que le transport exige, par conséquent, un temps plus long, il importe que le lait n'en souffre pas et qu'il arrive à la ville sans avoir subi d'altérations qui en rendraient la revente hasardeuse et la consommation pernicieuse pour la santé.

Le transport par camion-citerne calorifugé reste possible, mais devient beaucoup plus onéreux au profit du ehemin de fer qui combine les avantages de la rapidité, du bon marché et de la masse plus grande transportée.

C'est pourquoi se sont créées, à Paris notamment, des Messageries Laitières qui disposent d'un certain nombre de gares spécialement aménagées en vue de la réception du lait et exclusivement réservées à la manutention de ce genre de marchandise.

Ces gares laitières sont généralement divisées en deux 
départements superposés : le quai de réception et la salle de trans vasement.

Il n'y est procédé à aucun traitement du lait, celui-ci étant, dans la plupart des cas, filtré et pasteurisé au départ des centres de production.

Leur outillage est par conséquent réduit à un minimum. Le quai de réception, abrité sous un auvent, ne comporte, en effet, qu'une série de grands entonnoirs dans lesquels on déverse les cruches amenées par wagon, ou bien des têtes de conduite auxquelles on abouche le tuyau de décharge des wagons-citernes.

Quel que soit le procédé de déchargement utilisé, le lait est ainsi immédiatement entreposé dans de grandes cuves en acier vitrifié munies d'un agitateur destiné à éviter la montée de la crême. Ces cuves occupent la partie supérieure de la salle de transvasement, près du plafond.

De la paroi inférieure de chacune de ces cuves se détache un long tuyau souple, comparable à celui des distributeurs à essence, au moyen duquel un ouvrier remplit les cruches alignées sur le sol, en circulant dans des passages ménagés entre elles. Préalablement à cette opération, les cruches sont lavées et stérilisées par les procédés habituels, à la gare même.

Mais il faut noter que l'opération du remplissage des cruches se fait très rapidement, puisqu'il suffit de passer successivement de l'une à l'autre en tenant l'embout du tuyau souple au-dessus de chaque ouverture; il est, par conséquent, nécessaire que les cruches soient disposées et alignées en bon ordre dans la salle de transvasement avant l'arrivée du train laitier, la stérilisation et la manutention de quelques centaines de cruches demandant, malgré tout, un certain temps.

Ceci nous suggère, du point de vue hygiénique, quelques remarques qu'il nous paraît utile de signaler.

Nous avons, en effet, observé au cours d'une visite à une gare laitière, qu'à 4 heures de l'après-midi toutes les cruches étaient déjà stérilisées et alignées en bon ordre, prêtes à recevoir leur contenu, c'est-à-dire l'orifice ouvert, le couvercle, enlevé, pendant à sa chaîne de fixation. Or le train ne devait entrer en gare qu'à 7 heures du soir.

Pendant trois heures au moins, les cruches stérilisées étaient done exposées à la pénétration des poussières, les larges baies donnant sur le quai de chargement étant toutes ouvertes, en attendant l'arrivée des voitures de livraison.

Il se fait aussi que ces gares laitières sont situées dans la ville même et dans des quartiers fort animés puisque voisins d'une gare ou d'une ligne de chemin de fer. Dans celle, entre autres, qu'il 
nous a été permis de visiter, la salle de transvasement, largement ouverte comme nous l'avons déjà dit, était distante d'une trentaine de mètres d'une chaussée fort importante où la circulation était très intense.

Nul n'ignore que les poussières peuvent être transportées, par voie aérienne, à des distances parfois grandes et que ces distances augmentent d'autant plus que les grains de poussière sont plus ténus, et l'on sait également que les grains de poussière sont les véhicules certains de germes de toute nature et en particulier de germes pathogènes, notamment celui de la tuberculose provenant d'expectorations émises sur la voie publique. La plupart de ces germes gardent leur virulence même desséchés et cela est surtout vrai pour le bacille de Koch.

Nous nous sommes demandé, dans ces conditions, si une organisation semblable et, en ordre principal, la préparation trop longtemps à l'avance des cruches, ne constituait pas une erreur de technique et si le lait pasteurisé, amené grâce à des moyens de transport déjà perfectionnés, ne perdait pas ainsi toutes les qualités que l'on s'était efforcé de lui donner à la source et dont la moindre n'est pas son innocuité microbienne relative.

A quoi bon pasteuriser un lait si on l'expose à être réensemencé à un stade ultérieur de ses tribulations, comme il doit l'être certainement dans les cruches que nous avons $v u$ ainsi librement exposées aux poussières de la rue pendant un temps prolongé.

Tout mal porte généralement son remède et nous croyons qu'il en existe un, d'application facile, à la situation que nous venons de décrire.

Pourquoi, au moment où on range les cruches dans la salle de remplissage, après leur stérilisation, ne poserait-on pas simplement le couvercle sur l'orifice, sans l'enfoncer, mais de manière à ce qu'il recouvre cependant l'ouverture.

Il suffirait alors à l'ouvrier de soulever légèrement le couvercle pendant qu'il maintient l'embout du tuyau souple au-dessus de l'ouverture. Aussitôt la cruche pleine, il pourrait enfoncer le couvercle comme il doit l'être normalement pour réaliser l'obturation définitive de la eruche.

On ne peut évidemment exiger, qu'après la stérilisation, les couvercles soient placés normalement sur les cruches, c'est-à-dire enfoncés complètement. Il est à craindre, en effet, qu'au moment du remplissage, l'ouvrier ne rencontre des difficultés pour enlever les couvercles qui, enfoncés à frottement dur, ne se laissent pas toujours retirer avec aisance, d'autant plus que l'absence de contenu ne contribue pas à immobiliser la cruche sur le sol. L'ouvrier ayant une main déjà occupée par le tuyau de remplissage, verrait 
son travail ainsi compliqué et subirait de ce fait un retard appréciable, à moins qu'un aide n'ait d'autre occupation que de déboucher les cruches au moment voulu.

On ne pent perdre de vue cette exigence qui a force de loi en laiterie: c'est que tout ce qui touche au lait et le lait lui-même doivent être traités aseptiquement, tout comme les instruments dans une salle d'opération ou le matériel d'un laboratoire de bactériologie.

Le lait est un excellent milieu de culture, sinon le meilleur de tous. Une souillure minime dans un lait propre transforme rapidement celui-ci en un véritable bouillon de culture aussi dangereux que souvent insoupçonné,

En aucun cas, le mode de transport ne peut être une occasion de contamination du lait, ou mieux encore de surinfection, puisqu'il n'en subit déjà que trop à la source.

Mais, quand les dangers de contamination à la source sont annihilés, ou réduits dans leurs conséquences, par la pasteurisation, comme on est amené à le faire pour le lait destiné à être transporté à longue distance, la nécessité d'une protection efficace devient absolument impérieuse dans la suite.

C'est pourquoi, pour en revenir au système des gares laitières, nous estimons que la simple précaution de couvrir l'orifice des eruches fraîchement stérilisées au moyen du couvercle, même sans l'enfoncer complètement, constitue une mesure de sécurité suffisante mais nécessaire contre des contaminations pour ainsi dire fatales dans les conditions que nous avons pu observer.

Cette méthode n'entraîne aucune perte de temps, elle n'exige aucun appareillage coûteux et contribuerait certainement à maintenir le lait de province dans un état acceptable quant à sa teneur microbienne au moment de la consommation.

Bien plus, nous pensons que si la gare laitière se justifie comme une étape nécessaire dans le transport du lait à grande distance, elle n'est logiquement acceptable que comme une partie intégrante d'une laiterie, c'est-à-dire de l'usine même de traitement du lait.

En d'autres termes, les laiteries susceptibles d'avoir à traiter du lait provenant de régions éloignées devraient être directement raccordées à la voie de chemin de fer; cette disposition permettant un double gain: en temps et en sécurité.

En effet, la gare laitière isolée peut avoir une double destination : ou bien être le point de départ du mode de distribution à la cruche par le détaillant lui-même, ou bien servir à l'approvisionnement des laiteries urbaines.

Dans le premier cas, le lait, généralement pasteurisé au départ (laiterie productrice ou locale), risque d'être contaminé secondairement à l'occasion d'une manutention supplémentaire qui l'expose 
d'une manière intempestive aux poussières de la gare laitière et lui fait perdre, par conséquent, tout le bénéfice de la pasteurisation initiale.

On doit admettre, et rien n'est plus indispensable, qu'une fois pasteurisé, le lait ne peut plus prendre contact avec l'atmosphère extérieure jusqu'au moment de la livraison au consommateur.

Dans le second, cas, on ne peut que déplorer un transvasement superflu, avec tous les risques de contamination et la perte de temps qui l'accompagnent, et bien plus encore la nécessité d'une nouvelle pasteurisation à la laiterie urbaine pour pallier à ces risques inévitables.

Ces quelques considérations nous permettront de conclure en disant que les plus belles réalisations techniques ou industrielles ne peuvent échapper au contrôle hygiénique et que, bien souvent, bon nombre de détails doivent lui être sacrifiés, dans l'intérêt de la santé publique.

\title{
CONTRIBUTION A L'ÉTUDE DU LAIT DE BUFFLESSE Production, propriétés et composition, dérivés \\ par
}

\author{
J. Y. PISSAREWSKY \\ Docteur ès Sciences
}

Lauréat de l'Académie d'Agrieulture de France

\section{(Suite) \\ 2。 LES QUALITÉS ET LA COMPOSITION DU LAIT DE BUFFLESSE}

\section{A. PROPRIÉtés PHYSIQUES}

Couleur. - Le lait de bufflesse est de couleur nettement blanche neigeuse : même après un repos prolongé. il ne présente jamais dans sa couche superficielle la teinte jaunâtre que l'on rencontre dans le lait de vache riche en graisse. Cela est dû au fait que la matière grasse du lait de bufflesse est plus riche en stéarine, ce qui lui prête une couleur plus claire que celle de la matière grasse du lait de vache. L'opacité du lait de bufflesse ne disparaît pas après l'addition d'une forte quantité d'eau, ce qui incite souvent les commerçants à la fraude, car ce lait présente difficilement la teinte bleuâtre, même après un fort mouillage.

Odeur et saveur. - On a parfois reproché au lait de bufflesse de présenter une odeur de musc, et nous en dirons la raison dans un instant. Affirmons tout de suite avec la plus grande énergie que, 